\title{
11-th Balkan Congress of Otorhinolaryngology, Head-Neck Surgery May 31 - June 3, 2018, Varna, Golden Sands, Bulgaria
}

\section{Abstract}

Резюмета

\section{The Impact of the Nasal Trauma in early school-age and adolescents on the Development of the Nose in Future}

\author{
Author: Gabriela Kopacheva-Barsova \\ ENT University Hospital, University Campus "St. Mother Theresa”, Skopje, Macedonia
}

To prevent and to treat nasal trauma in children properly, because it can lead to displacement or depression of the nasal bones or septum. Second, our aim was, for the patient to recognise and create a mature decision for eventual nose changes which will be made with the operative intervention or they are not mature enough and the decisions were made by their parents.

Our retrospective study was made at University Clinic for Ear, Nose and Throat, Faculty of Medicine, Ss Cyril and Methodius University of Skopje in the period of 10 years (2008-2018). Seventy-three patients were admitted with recent or previous nasal trauma or nasal deformity. The first group of 32 were children and adolescents from 6-14 years old who were admitted to our hospital because of recent nasal trauma. The second group of 41 children and adolescents from 6-14 years old were admitted to our hospital because of previous nasal trauma, which was not treated on time, or it was not treated properly. They were admitted to our clinic for surgical intervention septo/rhinoplasty. The second group of patients fills the brief psychological questioner prepared by Clinical psychiatrist from University Clinic of Psychiatry, in Skopje, and their psychological reactions were taken into consideration.

Eleven of the children and adolescents who had nasal fracture without dislocation, who have no symptoms, minimal swelling, and no septal deviation or hematoma, were observed with a specific follow-up: 3 days after nasal fracture, then every week in the first month, after 1 month, and after 3 months period. Sixteen of children and adolescents who had a nasal fracture with subluxation of nasal septum were operated with closed reduction (repositio nasi) under general anaesthesia. The others with septal hematomas and subperichondrial abscess were treated as in adults' patients. The second group of 41 children and adolescents from 6-14 years old consisted with with the previous nasal trauma which was not treated on time or it was improperly treated. In $24(58.54 \%)$ of these patients septoplasty was performed and in $17(41.46 \%)$ was performed rhino septoplasty.

Often, difficult septal deformations in children are followed with deformation of the nasal pyramid (rhino scoliosis, rhino lordosis). In those cases, we cannot solve septal pathology without nasal pyramid intervention in the same time and opposite. Clinical reports have not produced solid evidence for the statement that septal surgery has no negative effect on nasal growth or can serve for correcting abnormal growth. The functional and esthetic problems of the patient, however, mean a continuous stimulus for further clinical and experimental investigations. 


\title{
Different Types of Periferal Vertigo
}

\author{
KUCI ANJEZA, MD, ENT, Center Policlinic Nr., Tirana, Albania \\ FEJZA JETMIRA, PhD, ENT, Mother Teresa Hospital, Tirana, Albania \\ BOCI BESIM, Prof.Ass, Head of ENT Department, Tirana, Albania
}

B.P.P.V or benign paroxysmal positional vertigo is one ofthe most common disorder of the inner s ear vestibular system, with two main pathologies: cupulolithiasis and canalolithiasis. The fispathology reaction is the migration of the otoconia into the semicircular canals or its fixation into the cupula, most of the cases in the posterior semicircular canal because of its anatomic construction.

Menier s desease or endolimfatic hydrops is a qualitative electrolytic disorder between the perilimf and endolimf causing a disorder in the osmotic pressure.

There are two main tipes of vertigo: in the B.P.P.V: breaf, spinning episodes of vertigo related to the position of the head in the space and the Menier s desease vertigo: long lasting episodes, with tinnitus, decrease hearing level and vegetative symptoms, mainly in the late faze.

There are patients that manifest both of these two deseases in the same time and theirclinic situation gets worse.

1. distinguish two tipes of vertigo in patients with Menier s desease and B.P.PV2.performing the repositioning maneuvers together with the medical therapy for these patients3.improving their life quality by curing correctly from the spinning vertigo of B.P.P.V and enlarging the period between two crisis in the Menier $\mathrm{s}$ desease

We have analyzed 30 parients from Mother Teresa Hospital and the Policlinic of the Specialities nr.3, Tirana, Albania, diagnosed with Menier s desease ,5 of them diagnosed with B.P.P.V as well. We have performed otoscopi, audiometry, vestibulometry and caloric tests, positional/positioning Dix- Hallpike to assure the diagnose and we found out the presence of B.P.P.V and Menier s desease in 5 cases and Menier s desease in 25 cases. We have done the repositioning maneuver in 5 patients with B.P.P.V, depending on the semicircular canal affected and prescribed the medication for all patients depending on the severity of the symptoms and performed the intratympanic gentamicin injections in some of them.

In all the cases with B.P.P.V and Menier s desease, that were the most complaining of all the patients after performing the repositioning maneuvers the breaf, spinning vertigo episodes disappeared and they went on with the Menier s therapy. For all the other cases we performed the medication therapy and intratympanic gentamycin injections.Our patients were satisfied after both these therapies and their quality of life improved.

\section{Surgical Treatment of Tympanosclerosis: Our results.}

\author{
Selis Gülseven Güven, Cem Uzun \\ Trakya University Faculty of Med., ORL\&HNS Dept., Edirne, Turkey
}

To investigate the post-operative clinical and audiological findings of patients with tympanosclerosis who were undergone surgery in our clinic.

We analysed data of the 750 patients who were undergone chronic suppurative otitis media surgery by the same surgeon (CU) between January 2003 and January 2018. Fiftyfive of them were operated only for tympanosclerosis. Follow-up records were available in 39 of

thosepatients. We evaluated otoscopic and audiological findings and compared them regarding the peroperative findings.

The mean follow-up time was 39 months. All of the tympanic membranes were intact (\%100). There was no perforation in any of the patients. Only one of them had myringitis on the anteroinferior part of the tympanic membrane. The mean air-bone gapsignificantly improved ( $34 \mathrm{~dB}$ vs $25 ; \mathrm{p}=0,001)$. The mean hearing also significantly improved after surgery $(51 \mathrm{~dB}$ vs $40 \mathrm{~dB} ; \mathrm{p}<0,0001)$. Regarding the tympanoplasty type, 
hearing results in type I and type II were significantly better after the surgery. There was no death ear. Only in one patient, the bone conduction worsened $10 \mathrm{~dB}$. Hearing became better more than $10 \mathrm{~dB}$ in $22(\% 56)$ patients.

Tympanosclerosis surgery with our technique results close tympanic membrane in all patients. Better hearing can be achieved in more than half of the patients. However, hearing results are not satisfactory in patients who are required type III tympanoplasty.

\section{Frontal sinus- type III Drafdrainage by surgical treatment failure- clinical cases}

Dzhambazov K.

Medical university - Plovdiv, ENT department University hospital "St. George" - Plovdiv

Frontal sinus disease has been difficult to address by endoscopes because of narrow frontal sinus ostia, superior location, and anatomic variations which limit visualization. The surgical options for frontal sinusitis range from limited opening of the anterior ethmoid to bilateral transseptal openings of the frontal sinus. The choice of the adequate strategy depends on individual anatomy, pathophysiologyof the disease and the aims of the surgery. In 1991 Draf suggested the first classification of endoscopic endonasal approach to the frontal sinus, known as fulda concept, which is considered to be the crucial point in the treatment of most frontal sinus deseases.

In the past three years, we performed a Draf III procedure on ten patients. After they had all been treated extra- and endonasally for several times without any clinical results, we applied the Draf III procedure to them. In this study, we will present two of these cases.

Indications for surgery included one osteoma, which had been surgically treated endonasally twice and once extranasally and one chronic allergic rhinosinuitiswith polyposis, which had been treated endonasally twice and extranasally three times for the last ten years. After the patients have undergone Draft III procedure and have been followed up endoscopically for six months, they showed an obvious neo-ostium and were clinically better but incompletely asymptomatic.

Draf III procedure is efficient and secure for patients who have failed convetional frontal sinus procedures and an appropriate alternative to frontal sinus obliteration. This technique provides good results in combination with minimized postoperative care and morbidity.

\section{Rare Manifestation of Gout - Gouty Tophi in the Nose}

\section{Georg IIliev, Polina Ivanova, Plamen Nedev}

Gout is a disease characterized by abnormal metabolism of the uric acid, leading to undesired buildup of monosodium urate crystals affecting mainly the first metatarsophalangeal joint of the foot and less commonly other joints or the soft tissues of the body. The prevalence increases with age, and it is higher among men than women.

To present an overview of the disease based on the literature and our clinical experience with this unusual manifestation of gouty tophi in the nose.

We performed a literature research in PubMed to identify unusual manifestations of gout and analyse the available data worldwide. We present the case of a 62-year-old male patient diagnosed with gout 20 years ago. He came to the clinics with a nasal hump and growing mass in the nose since 3 years presenting as nasal obstruction and deformity.

Regardless of the treatment with allopurinol and NSAIDs surgical treatment was necessary and the patient was taken to surgical resection of the mass in the nose. The histopathology revealed deposits of urate crystals surrounded by a granulomatous inflammation and confirmed the diagnosis - gouty tophi in the nose. In the follow-up period the patient reported improved breathing and aesthetic results. 
Tophi can be found in atypical locations, which increase morbidities and deformities caused by the disease. We report an interesting case of tophaceus gout, a rare manifestation of the disease with atypical location of tophi - in the nose.

\section{Endoscopic sinus surgery - four hands technique}

Zlatanov Hr., Milev S. - Department of Otorhinolaryngology - Military Medical Academy-Sofia Minkin Kr.- Department ofNeurosurgery - UMBAL "St. Iv. Rilski" - Sofia

To present the technique of endoscopic sinus surgery performed by a team of two surgeons for the management of skull base tumors.

Retrospective analysis of 8 cases of patients who had tumors of the nose, sinuses and skull base and were treated in the clinics with four hands endoscopic surgical techniques in the span of one year. Comparing the pre- and postoperative status of the patients.

In the span of one year we used the technique to treat two cases of CSF leak, three cases of pituitary adenoma and three cases of SCC of the paranasal sinuses.

The four hands technique for endoscopic sinus surgery allows for extended and complex sinus and skull base resections, as well as work in a team with neurosurgeons in cases of pituitary adenomas.

\section{Evaluation ofnasal mucociliary clearance time in cotton industryworkers}

Gül Soylu Özler, MD

Mustafa Kemal University, Department of

Otorhinolaryngology, Hatay

The aim of this study was to investigate the effect of cotton dust on nasal mucociliary clearance time in cotton industry workers.

A total of 50 ( 25 male, 25 female) subjects were included in the study. All of the subjects were non smokers. Nasal mucociliary clearance time of 50 cotton industry workers were measured and compared with the results of 50 age and sex matced control group. Also, the

correlation between nasal mucociliary clearance time and duration of exposure to cotton dust in cotton industry workers were evaluated.

The mean mucociliary clearance time in control group and cotton industry workers were $12.98 \pm 1.28$ and $17.88 \pm 3.64$ respectively. The mean mucociliary clearance time in cotton industry workers were significantly longer than control group ( $\mathrm{p}<0,0001)$. Also, there was positive correlation between duration of exposure to cotton dust and nasal mucociliary clearance time in wood industry workers $(\mathrm{r}=0.787, \mathrm{p}<0.0001)$.

The clinicians must keep in mind that mucociliary clearance time in cotton industry workers is prolonged. So the cotton industry workersmust be followed up more closely for respiratory tract diseases, chronic rhinosinusitis and lower airway infections.

\section{Case presentaion (st. post rhinoseptoplasticam.oedema baseos nasi.l.sin.)}

\section{Dr. Marinela Malinova,}

Prof. D-r. Miodrag Vrchakovski

Many patients are visiting ENT practices for a consultation or an advice on how to resolve a problem considering a difficulties with the functional breathing trough the nose because of the D.S.N. or other reasons, or are interested in a solution for better esthetics of the nasal pyramid. But there are patients who are coming because of the complications that occur after the surgical intervention of the nose. That kind of patients are bigger challenge for further diagnostic and if possible resolving of the problem. 
Female patient age 44 visited my office with anamnestic data that very often from the left side of the root of the nasal pyramid, soft, painless oedema occurs.If she does a mild pressure or massage from the external side than the oedema is getting smaller but after a while is coming back again. In a childhood she had an injury, a trauma of the nasal pyramid caused by an elbow blow during a gym class. That trauma was followed with severe pain which had remained for several weeks after, and with severe epistaxis that had stopped after 20 minutes. During that time an ENT examination was not performed. As time went by difficulties with nasal breathing occurred. Several years following the injury a consultation with ENT specialist was made and surgical intervention was advised because of the D.S.N. But because the patient was also unsatisfied with the esthetics, rhinoseptoplasty was performed at the age of 25. Data from the intervention was not available. The problem with the functional breathing was solved but as the time passed by the esthetic moment was not satisfactory because at the root of the nose subcutaneous, bone fragments are spotted that elevated the skin and the root of the nose does not look very good. Later on an oedema started to occur on the left side of the nasal base, sometimes spreading under the left eye.

ENT examination: Otoscopy: Both MAE and m.tympani:normal findings. Fibernasopharyngoscopy: slightly oedematose mucosa in cavum nasi, with normal coloring and normal moisture of the mucosa, good air flow of the both nostrials with no signs of inflammation. On inspection and palpation of the nose, on the left side of the nasal base: small, softie oedema can be spotted, without hyperemia, painless on palpation. On the dorsum nasi there are tiny bone fragments that can be spotted and palpated. (See photo) Oropharyngoscopy and Audiometry: Normal findings.

Status post rhinoseptoplasticam, with postoperative defect on the nasal pyramid, with multifractured and deformed bone base into several small bone fragments. There is external soft tissued oedema on the left side of the nasal base. PNS all with normal findings. Extracranial occipital on the right side there is osteoma on the large base with dimensions $25 \times 10 \mathrm{~mm}$ (Photo in attachment).

But from the CT scan it couldn't be defined what really happens and how that soft tissued oedema is forming and than with presassure obviously there is a drainage pathway and the oedema is getting smaller?

A suggestion came from Prof.D-r. M.Vrcakovski who is an eminent professor in the field of radiology that MRI and 3D CISS should be done in order to resolve this situation.

On the level of the nasal base left side subcutaneous there is a cystic formation fulfilled with a thicker liquid with characteristics of mucocella, sharply defined from the surrounding area with diameter of $9 \mathrm{~mm}$. This mucocella elevates the skin on that level. The finding indicates on mucocella posttraumatica. The rest MRI findings are normal.(Imaging photo in attachment)

The communication of the mucocella with cavum nasi was also detected so the situation how the cyst is getting full and than can be drainaged with a mild preassure was also explained. Because of the defect of the bone tissue in the space between processus pallatinum maxillae, processus nasalis ossis frontale and lamina cribriformis, there is a promination of the nasal mucosa into that space and a forming of one prolabsed cystic formation. That cystic formation is made of nasal mucosa which contains glands that are secreting and therefore that sacculus is filling with liquids that forms the soft tissued oedema on the left side nasal base. And than with the mild preassue or massage as the patient has done the liquid is draining into the nasal cavity. Because of the slight hypertrophy of the mucosa of the cavum nasi in that area ,that communication coulden't be identified by endoscopic examination.

Toillete of the nasal cavity with Sol. $\mathrm{NaCl} 0,9 \%$ was done (with suggestion to continue at home every day on regular basis once/twice per day)

Caps.Serrapeptase S.1x1/10 days in the morning before the breakfast (in order to help for better drainage) An advice and the next appointment was set up for 10 days later.

After 10 days the oedema was significantly reduced.

At the end when we already knew the reason the patient was suggested to make a consultation with a plastic surgeon for a surgical treatment which she has denied because as she said the purpose of her visit was the fear of some serious condition. When she found out that it was not serious ,overwhelmed from the exact diagnosis she has decided the situation ought to stay in status quo. 


\section{The role of otorhinolaringologist and dentist in the field of sleep medicine. Are we respecting the physiology?}

M. Milkov M., Ch. Madjova, J. Ruseva, P. Nedev, S. Gradanska, G. Iliev, M. Bliznakova,

N. Raichanova, A. Donchev, P. Petrov

Obstructive sleep apnea is a common disorder in adults and children worldwide, and, most probably, in Bulgaria as well. Recently, considerable advances in the clarification of its intimate pathophysiological mechanisms have been achieved. Proper and timely diagnosis and management of this pathology obligatorily require united efforts of interdisciplinary teams including specialists of different medical disciplines.

The objective of the present study was to summarize the ten-year experience gained by a group of otorhinolaryngologists, dental physicians, cardiologists, ophthalmologists, dieticians, physiotherapists and health care managers in diagnosing and treating the patients with obstructive sleep apnea of different severity from the North Eastern Bulgaria. Between January 2008 and March 2018, a total of 915 subjects complaining of sleep breathing disorders were covered. Of them, there were 545 males, 215 females and 155 children. Airway obstruction type was assessed by a variety of routine rhinolaryngological and dental-medical methods. Additionally, sleep features were monitored by polysomnography, polygraphy and ApneaGraph apparatus. Treatment with radiofrequency thermotherapy and coblation was applied. Soft palate somnoplastics was performed in 255, inferior hypertrophic concha reduction - in 285, nasal septoplastics - in 212, and tonsillectomy - in 45 patients. The rest 118 patients were forwarded to pulmologists in order to undergo CPAP management. This device widely approved for obstructive sleep apnea treatment was used by 23 patients following surgery, too. Intraoral appliances were prescribed to 85 patients after surgery and in 202 ones presenting with a slight form of the disease.

We could draw the conclusion that the complex interdisciplinary approach in snoring and obstructive sleep apnea represents the only effective option for these patients.

\section{Audio vestibular medicine. Hearing and diabetes mellitus}

\section{Milkov, Ch. Madjova, K. Hristozov, V. Jotova, M. Bliznakova, A. Donchev}

Global sugar consumption has dramatically increased in the past decades in many countries and in Bulgaria as well. Its abusive intake is responsible for peripheral insulin resistance, which causes diabetes mellitus and other components of the socially-significant metabolic syndrome such as obesity, arterial hypertension, and coronary artery disease. There is evidence that glucose metabolism has a significant impact on inner-ear physiology. Hearing impairment and type 2 diabetes mellitus are both highly prevalent disabling conditions, especially in younger subjects. Vestibular dysfunction and sensorineural hearing loss are common complications of diabetes mellitus.

The purpose of the present study was to objectify the subclinical vestibular disorders in type 2 diabetes mellitus.

Our 30 type 2 diabetes mellitus patients were divided into two groups according to the examination terms prior to and since 2010 onwards. All of them underwent at least two complex otorhinolaryngological, otoneurological and endoscrinological examinations as well as posturography, videonystagmography, pure tone audiometry, routine blood tests and glucose-loading tests each. There were no pathological finger-to-nose tests at all. In 26 patients (in 86,67\% of the cases), videonystagmography detected latent nystagmus aberrations. In 22 patients (in $73,33 \%$ of the cases), there were pathological Romberg,

Babinski-Weil and Fukuda tests. Since 2017 onwards, video head-impulse test was used along with videonystagmography in nine patients as well as in 69 healthy individuals within epidemiological screening. Our results identified the vestibular diabetic neuropathy as the primary disorder in this contingent. It presented with local demyelinization of vestibular nerve fibres and axonal loss. Eventual subsequent remyelinization could lead to local nerve deficit. The patients with reduced glucose tolerance and non-diagnosed type 2 diabetes mellitus were exposed to elevated risk of coronary artery disease, peripheral vascular disease and stroke as well because of microvascular or macrovascular pathology. 
In conclusion, it should be emphasized that modern broad-range otoneurological armamentarium is capable of suggesting the clinically latent vestibular damage in type 2 diabetes mellitus and its wide application should be recommended.

\section{Round Table Discussion: New biomaterials and implants used in the head area}

G. Marinov, K. Hörmann, C. Uzun,

Prof. A. Schmelzel M. Stankovich,

H. Grupcheva, S. Peev, P. Ruev, T. Karchioglu,

P. Dimov, M. Milkov

Modern biomaterials and implants used recently in the fields of otorhinolaryngology, ophthalmology and dental medicine meet the strict requirements for biostability, biocompatibility, biotolerability and absence of allergy-provoking factors as well. However, validation of a given biomaterial is time- and cost-consuming and, unfortunately, not always successful. Biomaterials of any kind are designed and manufactured for a precisely specified location in the human organism.

The main objective of the present round table discussion consists in the intensive opinion exchange of single participant's experience gained with biomaterials and implants and in the illustration of the solutions in complicated cases when a given biomaterial and/or implant has been rejected.

\section{Giant Esophageal Polyp: analysis of a surgical strategy}

Aleksandar Zlatarov, Nikolay Sapundzhiev,

Valentin Ignatov, Georgi Todorov, Dilyan Petrov, Anton Tonev, Deyan Dzhenkov, Nikola Kolev,

Krasimir Ivanov

Benign esophageal polyps uncommonly may progress to monstrous dimensions with relatively mild symptomatic. Scanty reports describe different approaches for surgical treatment. We present a case of a 65 -yearold male patient with a $14.5 \times 5.5 \times 4 \mathrm{~cm}$ fibrovascular esophageal polyp originating from the hypopharynx. The diagnosis was based on endoscopy, computer tomography, magnetic-resonance imaging, and virtual angiography. No major feeding vessel could be detected despite the significant dimensions of the mass.

Resection was performed with a combined approach, involving direct transoral exposition of the pedicle with Weerda distending diverticuloscope, rigid and flexible endoscopy. Traction with a silicone loop further improved the manipulation of the mass. The polyp's pedicle was injected with sclerosant and resected with 5-mm laparoscopic Ligasure. The considerable polyp size did not allow for transoral extraction of the mass, so a minilaparotomy was

required. The postoperative course is with no signs of bleeding. Histological analysis of the specimen confirmed the absence of large blood vessels in the pedicle.

Based on the review of the literature and the presented case we may conclude, that in giant esophageal polyps it is unlikely to have large feeding vessels. Still when planning endoscopic surgery consideration should be given to possible ways of intraoperative coagulation.

\section{Otorhinolaryngological applications of ultrasound cavitation energy-based device - histological features}

Georgi I. Davidov, Petranka R. Encheva,

Hristo B. Popov, Nikolay R. Sapundzhiev

Ultrasound cavitation device is a surgical instrument which uses between 1-16 MHz frequency ultrasound waves that apply energy to dissect, cut and coagulate tissue with minimal bleeding facilitate surgery. It is 
widely used in almost all surgical areas such as gynecology, cardiothoracic surgery, urology, general as well as head and neck surgery. The aim of this study is to analyze the effect of Ultrasound device (D\&A Ultrasurg II) on different tissues in histological aspect.

A retrospective study was conducted using data from medical records of 9 patients in ENT department. All of them had undergone surgery with D\&A Ultrasurg II. The resected tissues included tonsils, skeletal muscles, bone tissue, hyaline cartilage, multi layered flat epithelium and fibrous tissue. They were sent for histopathological examination. The specimens were examined microscopically using a Scannink Aperio ScanScope AT2 device (Aperio technologies Vista, CA) and analyzed with Image Scope V12.2.2.5015 (Aperio). Measurements were carried out at $1 \mathrm{~mm}$ intervals along the resection line and average values were estimated and analyzed.

The thickness of the resection line in tonsils was $437,26 \mu \mathrm{m}$. The vessels have marked degenerative changes in endothelial and smooth muscle cells and erythrocytes in vessels. Nerve fibers showed vacuolar degeneration and impaired structure. Lymphoid tissue has marked crush artefact changes (leaky lymphocytes) with poorly limiting lymphocyte boundaries. Fibrous tissue was deformed with distorted folded collagen fibers with basophilia or eosinophilia. The multi layered flat epithelium demonstrated severe vacuolar changes and arteficial detachment from the basal membrane. Hyaline cartilage showed impaired structure and eosinophilia with no chondrocytes in the necrotic area. The analysis of skeletal muscles displayed distorted borders between transversally grown muscle cells and eosinophilia. Bone tissue sample did not allow histological assessment of changes due to decalcification.

The analyzed tissues and the histological examination demonstrated the lateral thermal damage of Ultrasurg II

\section{Videofluoroscopic follow up of the hypopharynx in laryngectomees after radiotherapy}

\section{Blagovesta Spasova, Nikolay Sapundzhev, Assen Assenov, Darina Ivanova, Boyan Balev}

Dysphagia is a common complaint after laryngectomy. It progresses after postoperative radiotherapy (PORT) and is associated with a decrease of the quality of life. The aim of the

study was to evaluate the changes in the neohypopharynx as a result of post-operative RT in laryngectomees by video fluoroscopy (VFS).

The charts and digital VFS images of patients who, were subjected to VFS in the early postoperative period after laryngectomy and later had had another VFS examination after PORT, were retrospectively analyzed. In the lateral projection the neohypoharyngeal dimensions were measured at the levels of the cervical vertebrae and at the segment with minimal lumen. The measurements were calibrated to the height of C3. The dimensions at the individual levels, their mean value and the dimensions and location of the narrowest lumen before and after RT, were compared.

A total of 16 patients with VFS before and after LT were identified (all male, mean age $60.5 \pm 7$ years). All were subjected to laryngectomy for advanced carcinoma $(5 \mathrm{xT} 3,6 \mathrm{xT} 4,2 \mathrm{xN} 2 \mathrm{c}, 1 \mathrm{xN1}, 8 \mathrm{xN} 0$.) with standard surgical technique. In 4 patients, the archived images didn't allow accurate analysis and they were excluded. The VFS was performed $4.4 \pm 7$ months after RT. In all patients changes in the size of the neohypopharynx were detectable after RT. In 7 (63.6\%) patients, a reduction in mean neohypopharyngeal size was observed with an average of $12.6 \%$. It $4(36.34 \%)$ there was an increase by $18.6 \%$ on average. In 1 patient $(6.3 \%)$ a neohypopharyngeal narrowing was observed at all levels by $40.5 \%$ on average. In 1 patient $(6.3 \%)$ an increase in neohypopharyngeal size was observed at all levels, averaging $20.5 \%$.

The VFS examination before and after RT in laryngectomees doesn`t detect a tendency for neohypopharyngeal stenosis. The worsening of dysphagia in such patients can hardly be associated with mechanical stenosis. 


\section{First 5 Years before IV-th Balkan Congress of Otorhinolaryngology, Head-Neck Surgery. Our Activities and Prof. Dr. T. Karchev}

Pavel Dimov

Professor of Otorhinolaryngology, Medical Faculty, Trakia University Stara Zagora, Bulgaria

The last 20 years have served as an important period of new scientific relationship among medical doctors at Balkan countries. Good international conditions at the end of the last century formed the basis to encourage mobility across borders for students, doctors and staff in Thrace to the Balkans.

Review of our first 5 years before 2004 IV-th Balkan Congress of Otorhinolaryngology, Head-Neck Surgery scientific activities and participation of Prof. Dr. T. Karchev

Our Continuing Medical Education (CME) in otology and scientific activities in the first 5 years before 2004 IV-th Balkan Congress of Otorhinolaryngology, Head-Neck Surgery were analyzed: 1999-2004 otology meetings ORL Clinic-Stara Zagora, relationships ORL Clinics Edirne, Ankara, Bursa; 2001 Athens 11-th Panhellenic Congress of ORL-HNS; ORL Nish, titles of scientific presentations and papers of Prof. Dr. T. Karchev are indicated

Nine otology and neuro-otology (ONO) scientific events were organized (3 International Seminars, 2 International Meetings, 3 Symposiums of St. Ivan Rilski, 1 Intensive summer

ONO Course); 2 Proceedings in Otology and Neurootology, 1 Proceedings - Prevention of Social Significant Diseases); 4 years "Pro Otology" BJONO; 3 years web-page www.prootology.domino.bg; 2 temporal bone dissection courses; Prof. Dr. T. Karchev short biography are presented.

All of these efforts have formed the basis for international scientific collaborations in the otorhinolaryngology field; 2001 was renovated the Balkan Society of Otorhinolaryngology and Head-Neck Surgery and 2004 was organized IV-th Balkan Congress of Otorhinolaryngology, Head-Neck Surgery

The first 5 years scientific meetings before 2004 IV-th Balkan Congress of Otorhinolaryngology, Head-Neck Surgery were the beginning and the base of renovation of the new Balkan ORL collaboration. In these 5 years scientific activities was included and participation of Prof. Dr. T. Karchev.

\section{Advantages of the Mastoid Cavity Obliteration in Treatment of Cholesetatoma}

Rouev $P$.

ENT Dept., Hospital TRAKIA, Stara Zagora,

Bulgaria

The primary goal in the surgical management of chronic otitis media with cholesteatoma is the creation of a dry, safe ear. The "gold standard" for management of cholesteatoma is following the matrix of cholesteatoma from anterior to posterior. After complete cholesteatoma removal if total canal wall reconstruction is not possible we make mastoid cavity obliteration.

Retrospective study of 121 patients with cholesteatoma were surgically treatment during 01.07.2014 - 31.03.2018 with mastoid cavity obliteration after partial canal wall reconstruction.

Behind complete cholesteatoma removal and after tympanoplasty with partial posterior canal wall reconstruction and mastoid cavity obliteration we have not significant early and late postoperative complications. Recurrence of the disease occurred in 9 patients (7.44\%). Only one patient with mastoid obliteration with Bioactive Glass granules developed postoperative granulation and will be revised - the granules must be fully covered with cartilage or connective tissue.

The mastoid cavity obliteration procedure prevent development of postoperative retraction pockets and isolating the tympanum from the attic and mastoid and in this way is a safe and effective technique for the rehabilitation of open mastoidectomies. Functional results of this technique is variable, but with the possibility of fitting an HA and implantation, and otherwise is well tolerated from the patients. 


\title{
Advantages of the Mastoid Cavity Obliteration in Treatment of Cholesetatoma
}

Rouev P. ENT Dept., Hospital TRAKIA, Stara Zagora, Bulgaria

The primary goal in the surgical management of chronic otitis media with cholesteatoma is the creation of a dry, safe ear. The "gold standard" for management of cholesteatoma is following the matrix of cholesteatoma from anterior to posterior. After complete cholesteatoma removal if total canal wall reconstruction is not possible we make mastoid cavity obliteration.

Retrospective study of 121 patients with cholesteatoma were surgically treatment during 01.07.2014 - 31.03.2018 with mastoid cavity obliteration after partial canal wall reconstruction.

Behind complete cholesteatoma removal and after tympanoplasty with partial posterior canal wall reconstruction and mastoid cavity obliteration we have not significant early and late postoperative complications. Recurrence of the disease occurred in 9 patients (7.44\%). Only one patient with mastoid obliteration with Bioactive Glass granules developed postoperative granulation and will be revised - the granules must be fully covered with cartilage or connective tissue.

The mastoid cavity obliteration procedure prevent development of postoperative retraction pockets and isolating the tympanum from the attic and mastoid and in this way is a safe and effective technique for the rehabilitation of open mastoidectomies. Functional results of this technique is variable, but with the possibility of fitting an HA and implantation, and otherwise is well tolerated from the patients.

\section{The Nasal Structure and Function in Rhinoplasty}

\author{
Frodita Jakimovska \\ University ENT Clinic, Skopje, Macedonia
}

The nose is the central feature of the face, and is the major determinant of facial beauty. Humps, asymmetry, tip deformities, deviated and broad dorsums can all be corrected and refined through a number of elegant surgical maneuvers. Rhinoplasty surgery is much like architecture. Nasal structure and function go hand in hand, and the challenge is creating a nose the looks fantastic and allows easy passage of air. The aim is to consider how the graduated surgical techniques for the correction of nasal pyramide and septal deviations could affect the nasal form and function and to evaluate the patient satisfaction of the aesthetic and functional outcomes. The review of rhinoplasty cases would undertaken to delineate the execution of specific surgical techniques in creating deprojection of nasal dorsum and straightening of deviated nose combined with tip refinement and projection. The outcomes of aesthetic and functional nasal surgery are difficult to assess objectively due to the intricate balance between nasal structure and function.

\section{The effects of silicone nasal septal splints and Polyvinyl alcohol sponge after septoplasty on postoperative pain and pain during pack removal}

\section{T. Ersozlu, A. Cakmak}

To compare silicone nasal septal splints with integral airway and a polyvinyl alcohol (PVA) sponge after septoplasty in terms of patient comfort (both with the pack in place and during removal) and postoperative complications.

This study involved 169 patients who underwent septoplasty without additional nasal procedures due to nasal septal deviation. They were allocated into two groups. Group A comprised 90 patients who underwent septoplasty and who were packed with silicone nasal septal splints. Group B comprised 76 patients who underwent septoplasty and who were packed with a PVA sponge. They were removed on the second postoperative day. Patients were asked to record pain levels using a visual analog scale (VAS).

The patients in the groups had similar mean ages: 29.77 years (range, $19-74$ years) and 23.77 years (range, 
21 - 37 years) in Group A and Group B, respectively ( $p>0.05)$. VAS scores were significantly lower in Group A than in Group B at the 1st, 2nd, 4th, 8th, 12th, 24th, 48th, and 72nd postoperative hours (p < 0.0001). There were significantly higher pain levels associated with PVA sponge pack removal than with silicone nasal septum splint removal (average pain scores: 3.57 vs. 1.99, respectively; $\mathrm{p}<0.05$ ).

Intranasal septal splints with integral airway result in less postoperative pain during removal without increasing postoperative complications; thus, they can be used as an effective alternative to PVA sponge packing after septoplasty.

Nasal septal surgery is one of the most frequently performed operations in otorhinolaryngology clinics $(1,2)$. Nasal packing is one of the effective tools for septal support and avoiding adhesion after septal surgery. In recent years in particular, silicone nasal septal splints with integral airway have been used to reduce pain and increase patient comfort postoperatively $(3,4,5)$. The usage of nasal packing after septoplasty may rarely cause life-threatening complications $(6,7,8)$, but nasal packing also prevents some complication such as adhesion formation, bleeding, hematoma, and septal perforation $(3,9,10)$. These complications may be minimized by using nasal packing after surgery and during postoperative care and choosing the best nasal packing option $(6,7)$. During or after septal operations, effective postoperative pain control is essential because pain may decrease the quality of life. The most common morbidity associated with packing is postoperative pain and pain during its removal $(2,3,4,5,9,10,11)$. Patients usually feel anxious about pain before surgery. Postoperatively nasal packing materials, such as vaseline gauze, glove fingers, Oxycel (Woundcare Ltd., Manchester, UK), Gelfoam (Pharmacia \& Upjohn, Michigan, USA), Merocel (Medtronic, Connecticut, USA), silastic sheets, fibrin glue, synthetic polyurethane foam, Rapid Rhino (Smith \& Nephew Inc., Austin, USA), and silicone intranasal splints, are used (2-7,10-12).

Suture techniques are applied without using nasal packing $(2,9,10,13,14)$. Worldwide, the type of nasal packing material to be used after septoplasty is unclear. Though the use of nasal splints in recent years has increased, comparative studies on them are not sufficient.

We aimed to evaluate silicone nasal septal splints with integral airway and the polyvinyl alcohol (PVA) sponge Merocel (Medtronic, Connecticut, USA) after septoplasty with regard to patient's pain both with the pack in place and during removal as well as postoperative complications.

This study involved 169 patients who underwent only septoplasty due to nasal septal deviation between July 2011 and March 2013. The study protocol was approved by Gülhane Military Medical Academy, Medical Faculty and Hospital Ethics Committee (05/07/2011-176). All patients were informed on surgical risks, possible complications related to the operation, and the research protocol before they signed the informed consent form. All patients were older than 18 years. Exclusion criteria were as follows: immunodeficiency or any sign of infection at the time of operation previous history of any type of nasal surgery, and presence of nasal polyps or chronic sinusitis. Patient selection for surgery was based on the clinical history, rhinology examination results, and nasal endoscopy findings. Preoperative laboratory evaluations included complete blood count, thrombin time, prothrombin time, and thromboplastin time.

The patients were divided into two groups: Group A comprised 90 patients who underwent septoplasty and who were packed with mupirocin cream (Bactroban 2\% cram, Glaxo Smith Kline, Istanbul, Turkey)-soaked silicone nasal septal splints with integral airway (DOYLE splint; Boston Medical Products, MA, USA) in both nasal cavities. It was removed on the second postoperative day.

Group B comprised 76 patients who underwent septoplasty and who were packed with mupirocin cream (Bactroban 2\% cream; GlaxoSmithKline, Istanbul, Turkey)-soaked PVA sponge Merocel (Medtronic, Connecticut, USA) with integral airway in both nasal cavities. It was removed on the second postoperative day.

All septoplasty procedures were performed under general anesthesia. They were conducted using a headlight. At the beginning of anesthesia, $20 \mathrm{mg} / \mathrm{mL}$ of lidocaine hydrochloride and $0.0125 \mathrm{mg} / \mathrm{mL}$ of epinephrine hydrochloride (JETOKAİNE $2 \mathrm{ML}$; Adeka, Istanbul, Turkey) were administered to all patients to aid homeostasis. A local anesthetic agent was infiltrated into the nasal septum bilaterally in the submucoperichondrial-subperiosteal plane. A hemitransfixion incision was made in all patients. Deviated osteochondral parts were removed, reshaped, and placed back again. All incisions were sutured using 3/0 polyglytone (CaprosynTM; Medtronic, Connecticut, USA) Both materials were packed in both nasal cavities. The 
patients were blinded to the type of packs used; however, the surgeons were not blinded.

All patients were given $1 \mathrm{~g}$ of intravenous cefazolin. Postoperatively, patients were given $500 \mathrm{mg}$ of paracetamol three times a day orally.

No other perioperative analgesic medication was used. To determine the level of postoperative pain, a continuous $10-\mathrm{cm}$ visual analog scale (VAS) was used, with " 0 " indicating no pain and " 10 " indicating the most severe pain. Patients were asked to mark the severity of their pain on the scale at predefined time points [at the 1st, 2nd, 4th, 8th, 12th, 24th, 48th, and 72nd postoperative hours and at the removal time (48th hour)], and scores were measured and recorded in millimeters.

Patients were given $500 \mathrm{mg}$ of paracetamol three times a day. The prescriber was blinded to the type of nasal packs used. Postoperative complications were recorded in both groups. All patients were followed up until the 20th postoperative day.

Raw data obtained from the questionnaire with application data were used to be converted and analyzed with the statistical software program IBM SPSS (Statistical Package for Social

Sciences, Virginia, USA) for Windows 20.0. Descriptive statistical analysis was performing using the mean ( ), standard deviation, minimum, frequency, and percentage. Research data were examined with the Kolmogorov-Smirnov test and graphical representation. Quantitative data were analyzed used Mann-Whitney U Test and independent sample $t$ test.The paired sample $t$ test and Wilcoxon test were used to analyze repeated measures. Results were evaluated in terms of $95 \%$ confidence interval and significance of $p<0.05$.

The silicone nasal splint group (Group A) included 90 patients and the sponge pack group (Group B) included 79 patients. Data of all patients were included in the statistical analysis. The patients in the groups had similar mean ages: 29.77 years (range, 19-74 years) and 23.77 years (range, 21-37 years) in Group A and Group B, respectively ( $p>0.05)$. All patients were symptom-free at the follow-up visit on the 20th postoperative day.

VAS scores were significantly lower in Group A than in Group B at the 1st, 2nd, 4th, 8th, 12th, 24th, 48th, and 72nd postoperative hours $(\mathrm{p}<0.0001)$ (Figure 1$)$. There were significantly higher pain levels associated with Merocel (Medtronic, Connecticut, USA) pack removal than with silicone nasal splint removal (average pain scores: 3.57 vs. 1.99, respectively; $\mathrm{p}<0.05$ ) (Figure 2).

Hematoma was observed in three patients in Group A and two patients in Group B. Further, in Group B, purulent discharge was observed in two patients. Hematoma was drained, and nasal packing was done for two additional days. Patients were treated with $1000 \mathrm{mg}$ of amoxicillin two times a day orally for seven more days.

All patients recovered by the 14th day. There was no nausea and vomiting-related difference between the groups. None of the patients presented any complaints related to pain, nausea, or vomiting in the follow-up visit on the 14th day postoperatively. There was no fever in any of the patients. There was no statistically significant difference between the groups due to postoperative complications $(\mathrm{p}>0.05)$. No cardiovascular or neurological complications were observed in both groups.

asal obstruction is a common problem and is usually treated with septoplasty. (15). Septoplasty is one of the most common operation in the field of otolaryngology $(1,2)$. Pain persists after all surgeries, and it may lead to difficulties for patients trying to return to their routine lives. Many patients' concerns about nasal packs used after septoplasty and pain during the removal of nasal packs $(2,9,14)$. However, in recent years, new surgical procedures and materials render the postoperative process more painless $(9,11,13)$.

Nasal packing is used after septoplasty to control postoperative bleeding, fixate septal cartilage, and reduce adherent mucosa and septal hematoma (3-6). However, nasal packing leads to problems such as pain, mucosal damage, blockage of the Eustachian tube, infection, and dry mouth $(2,3,9,10,14)$. Serious problems such as toxic shock syndrome, cardiac arrhythmia, and even death have become very rare with the use of antibiotics and comfortable packs and with close monitoring (9).

Worldwide, many types of nasal packs have been used, but a group of physicians used septal suture techniques in their studies instead of packing after septoplasty (2-15). Therefore, there is no consensus on what type of nasal packs need to be used after septoplasty. In our clinic, we used both silicone nasal septal splints with integral airway and a PVA sponge with integral airway after septal surgery; in this study, we aimed to compare the pain levels $72 \mathrm{~h}$ after the operation and at the time of removing the nasal packs. The most 
disturbing complaints have been reported to be at the time of removing of the pack and the postoperative period. Unlike other studies, we also evaluated pain at the 72nd hour. The VAS scoring system was used for evaluating pain scores. This method has high sensitivity and is easy to use (11). Further, we evaluated both groups in terms of postoperative complications. We determined no significant difference in complications between the two groups.

Merocel is one of the most commonly used and cost-effective nasal packs $(3,5,9)$. However, because nasal packs adhere to the mucosa, bleeding and pain during the time of removal create difficulties $(9,13)$. Other materials have been used to minimize these disadvantages. Hesham et al. conducted a study with Rapid Rhino and Merocel; they made comparisons in terms of pain and complications and found that Rapid Rhino is better in terms of relieving pain during removal and bleeding (12). Similarly, Series 500 nasal packs, synthetic polyurethane foam, gauze in glove finger, and FloSeal were found to be less painful than Merocel during the removal of nasal packs $(5,11,16-18)$. Acioglu et al. evaluated pain, nasal fullness, and bleeding potential associated with using four nasal packing materials (Merocel, Doyle Combo splint, Merocel clothed with glove finger, and vaseline gauze) and determined that Merocel had the highest pain potential during removal and the highest rate of bleeding following removal (11). In our study, we compared postoperative discomfort and pain scores between two nasal packing groups. Postoperative pain scores increased in group B (Merocel group), which was in agreement with findings in the literature $(11,12)$. Similar to the above studies, in our study, we observed that group A (nasal splint group) was better than Merocel in terms of reducing postoperative pain scores and pain during removal.

Asaka et al. compared silicone plates with sponge-like packing and found less postoperative pain scores in the silicone plate group (3). Jung et al. showed that silastic septal splints prevent postoperative adhesion and mucosal erosion and cause less discomfort in the early postoperative period; they also recommended the use of proper septal positioning (6). Aksoy et al. used internal nasal splints in their study; they compared postoperative early and late bleeding, septal hematoma, and adherent mucosa formation and found that complication rates do not significantly differ according to the splint removal time. The patients whose splints were removed at the first and fifth postoperative days had similar complication rates in the study of Aksoy and his colleagues. Lubianca-Neto et al. evaluated the rates of hemorrhagic complications after nasal pack removal when left for 24 and $48 \mathrm{~h}$. In their studies, the rate of postoperative complications did not differ according to the time of nasal packing $(7,19)$. As emphasized in the literature above, we removed both the PVA sponge Merocel with integral airway and silicone nasal septal splints with integral airway on the second postoperative day.

Yilmaz et al. determined that silicone nasal splints cause less Eustachian tube dysfunction (8). Septal suture is recommended as an alternative to packing. There are literatures reports that septal sutures is better in terms of postoperative pain and complications and that there is no need to use splints $(2,9,10,13,14,20-23)$. Some of these studies have been conducted before the 2000s; today, thin, flexible, integral airway, and comfortable silicone splints are used. In addition, Kula et al. found that that nasal packs did not affect mucociliary clearance (24). Özkırış et al. compared trans-septal suturing, nasal splints, and Merocel (Medtronic,

Connecticut, USA) and found lesser postoperative pain in the trans-septal suturing group than in the other groups; however, the surgery time was found to be longer in the trans-septal suturing group than in the other groups (14). There is a lack of consensus regarding the timing of nasal pack removal after septoplasty. Another group that had nasal packing removed the fifth day after the operation may be created to determine the effect of the duration of using the nasal packing on pain. Our study shows that nasal splints was better than the PVA sponge in terms of patient comfort. Therefore, silicone nasal splints can be selected for routine use for patient comfort in septal surgery.

Nasal packing is used for the reduction of postoperative complications and septal stabilization. After septal surgery, there is no consensus on the methods or the ideal pack to be used. We conclude that intranasal septal splints with integral airway result in less postoperative pain during removal without increasing postoperative complications; thus, they can be used as an effective alternative to the PVA sponge (Merocel) packing after septoplasty. 


\section{Nasal obstruction after rhinoseptoplasty}

Gabriela Kopacheva - Barsova

ENT University Hospital, University Campus "St. Mother Theresa" Faculty of Medicine, Ss Cyril and Methodius University of Skopje, Republic of Macedonia

The success of cosmetic rhinoplasty is initially judged by aesthetics; however, the final result is deemed successful only if the internal nose functions as well as the external nose appears. Surgeons must also appreciate the nose as a living form of architecture and adhere to these same principles. It is only by doing so that we will create both beautifully functioning and aesthetically pleasing noses.

The etiology of post-rhinoplasty nasal obstruction can be multifactorial but is primarily the result of the interplay between 2 factors: Unrecognized preexisting nasal conditions (deviated nasal septum, turbinate hypertrophy, mucosal disease), Decreasing in the nasal valve area after rhinoplasty are responsible for most cases of postrhinoplasty nasal obstruction. The prevalence of airway impairment after aesthetic rhinoplasty may be as high as $10 \%$.

The treatment of post-rhinoplasty nasal obstruction has no absolute indications. The extent of medical and surgical treatment depends on the severity of symptoms and the patient's desire to improve his or her ability to breathe through the nose.

Surgical correction of nasal valve obstruction is extremely effective in improving subjective nasal obstruction. Success of this procedure is predicated by correct diagnosis and appropriate surgical technique. In cases involving individuals who have previously undergone reduction rhinoplasty, there should be a high index of suspicion for nasal valve obstruction. Reduction of a high cartilaginous dorsum can lead to internal valve collapse, while tip reduction and aggressive cephalic trimming of lateral crura can result in external valve collapse. Cartilage grafts used as spreader and alar batten grafts allow restoration of the nasal valve to a more normal anatomical shape. The cosmetic effect of such reconstruction is widening of the middle third of the nose, thereby achieving a wider appearance of the nasal tip.

\section{Five Years' Experience of Bone-anchored Hearing Aid (Baha) Application in Selçuk University Medical Faculty Hospital}

\section{Yunus Emre Topan, Bahar Çolpan, Turgut Çelik, Ömer Erdur}

We aimed to present our experience of bone-anchored hearing aid application in Selçuk University Medical Faculty Hospital in this study.

Material- Method: 19 patients who underwent BAHA surgery between 2013-2108 and whose one year follow-up was completed, were retrospectively reviewed.

The age range of patients was between $6-45$ years.11 of patients were female (\%57.9),8 of patients were male (\%42.1). 4 of patients were in pediatric age group (mean age 6), remaining 15 patients were adults (mean age 39.7). 1 patient of pediatric age group had Kleinfelter syndrome and was applied BAHA due to failure of previous tympanoplasty which was done because of ossicular chain erosion. 6 patients were operated for conduction type hearing loss due to aural atresia and remaining 12 patients were operated because of chronic otitis media. Air way hearing level was $69+11 \mathrm{~dB}$ preoperatively,bone way hearing threshould was $25+9 \mathrm{~dB}$. Free sound field BAHA threshould was $45+7 \mathrm{~dB}$ postoperatively.Non of our patients had intraoperative or perioperative complication. Two patients $(16.6 \%)$ who were followed-up at least for one year experienced implant loss due to local infection and excessive soft tissue hypertrophy surrounding the abutment. The most common complications were local infection and soft tissue infection in the implantiation area. Two patients (16.6\%) required recurrent antibiotic treatment. Revision surgery was applied for 2 patients who had complication and none complication developed during the follow-up period of 1 year.

Although the number of patients was low, the results of BAHA application were found to be quite satisfactory. 


\title{
Metastatic Tumors in the Neck With an Unknown Primary Tumor
}

\author{
Zorica Novakovic, Nebojsa Jovic, Marinko Karalic, Aleksandra Aleksic, Natasa Guzina Golac
}

Metastatic tumors in the neck with an unknown primary tumor is one of the most difficult problems in the head and neck oncology. They are a heterogeneous group of metastatic tumors in which primary localization can not be detected in a detailed history, careful clinical examination and extensive diagnostic procedures. Metastases to cervical lymph node cancer of unknown primary origin are represented between 3\% and 9\% in the total number of patients with head and neck cancers. In the five-year period (from January 2010. to December 2015.) in the Department of ENT, University Clinical Center of the Republik of Srpska Banjaluka, 42 patients were treated, of which three females and 39 males, mean age 52.71 years. Metastases were usually localized in the upper jugular chain. They dominated squamous cell carcinomas $88.1 \%$. The largest number of metastases was N2 category 57.14\%. Operable metastases in planocellulare cancer were treated by the method of radical and functional neck dissection and postoperative radiotherapy is applied.

Chemotherapy and radiotherapy were administered in patients with metastatic anaplastic or undifferentiated carcinoma. No sign of disease was at $42.86 \%$ patients who were monitored for average 50,72 months. 19 patients died, and were monitored for an average of 11,70 months.

Before setting up the diagnosis of metastatic tumors of unknown primary localization, and upon establishing the basic diagnosis, that did not detect the primary tumor, it is necessary to conduct more detailed research, the so called "step by step" diagnostic plan. 